\title{
Analysis of fitness furniture for college dormitory environment
}

\author{
Jun-qi ZHAO ${ }^{1,2}$,Ming $\mathrm{CHEN}^{1,2, a}$,Jian-hua LYU ${ }^{1,2, b, *}$ \\ ${ }^{1}$ Forestry College, Sichuan Agricultural University, Chengdu 611130, China \\ ${ }^{2}$ Key Laboratory of Wood Industry and Furniture Engineering of Sichuan Provincial Colleges and \\ Universities, Sichuan Agricultural University, Chengdu 611130, China \\ acchenming@sicau.edu.cn, ${ }^{\mathrm{b}} \mathrm{ljh} @$ sicau.edu.cn \\ ${ }^{*}$ Corresponding author
}

Keywords: college students, dormitory, fitness furniture.

\begin{abstract}
The fitness requirements of college students were expounded after analysis on the current situation in this paper. College students' dormitory is an important place for learning and living, where students spend lots of spare time. Therefore, in order to improve the physical quality of college students, this paper put forward the standpoint of using fitness furniture in the college dormitory. The environment of dormitory as well as its characteristics were analyzed and summarized. The furniture which can allow students to exercise in the course of their study and leisure was designed for verification with related researches, then an approach for the market-oriented fitness furniture design was developed. Finally, in order to adapt to the needs of society, we also evaluated the design of fitness furniture.
\end{abstract}

\section{Introduction}

College students are a group of persons with high quality in China, and they are main force of social development in the future. Their physical health status directly affects the value of college students to the whole society. The results showed that physical fitness, physical function and physical quality of them have decreased significantly in recent years. It is imperative to improve the physical quality of college students with their current health states [1]. Generally, the outdoor fitness of college students is often affected by the weather and air quality, however, indoor fitness can effectively avoid or overcome the disadvantages of outdoor fitness. How to effectively use indoor space to improve student fitness interest, as well as to extend the fitness time has become the focus of the future study. As a place for students to rest, study and entertain, students' apartment is undoubtedly the most important place for them. So providing space for fitness activities and positioning the apartment furniture will greatly extend the fitness time and improve college students' physical condition. In summary, it is essential for us to study the dormitory fitness furniture design.

\section{Indoor Fitness Condition of College Students}

Researches showed that physical fitness has not only the function of physical exercise and physical fitness, but also improving the negative mood such as anxiety and depression in various forms of fitness exercises to healthy people. On the existence of psychological issues, long-term physical exercise to eliminate bad emotions is more significant. Fitness can reduce the occurrence of unhealthy psychology and improve the vitality of life, so that body and spirit are in good condition. Therefore, the development of fitness for college students and a variety of leisure sports fitness method are urgent problems to be solved [2-3].

In order to improve the physical fitness of students, some colleges and universities had opened a number of fitness courses, but the results were unsatisfactory. For college students, the subjective individual reasons include the interest in fitness, human inertia, fitness, emotional experience, fitness awareness, no special projects, their own needs and other aspects; Objective environmental factors include facilities and equipment, campus sports culture atmosphere, personal guidance and leadership, weather environment, etc.[4].A survey represented that college students prefer to take 
part in interesting, practical, strong independent projects, and they pursue more colorful, variform of entertainment fitness [5]. They tend to choose a reasonable way and a good place to exercise, because only in that way, can they avoid the objective unfavorable factors, and improve the sense of subjective fitness.

The choice and use of fitness methods directly affects the effect of fitness, but also reflect the development direction and level of fitness. As for indoor fitness, its methods can be broadly divided into two categories, one of them is free fitness, such as aerobics, gymnastics, yoga and swimming; the other one is equipment fitness, such as dumbbells, barbells and other weightlifting equipment: spring rally, pulley rally, and other strength training equipment; power bicycles, flat run and other aerobic training equipment; as well as ball, bar and other light equipment such as gymnastics, etc.[6]. However, their incomes are the source and basis of consumption, for almost college students without income, the family economic conditions directly determine the consumer behavior of their fitness [7], thus only a few people can go to the gym for the system fitness training.

\section{Research on the Environment of Dormitory in Colleges and Universities}

Dormitory as a place for students to rest, study and entertainment, it is undoubtedly the place where they use the longest. In accordance with the survey, college boys spend an average of 82.4 hours in the dormitory and 92.1 hours for girls, accounting for $49.04 \%$ and $54.82 \%$ of the total, respectively. It can be seen that half or more of college life is spent in the dormitory[8].

Nowadays, higher education is paying more attention to people-oriented education concept. To keep students' best learning status and guarantee students' healthy growth, the space of a college dormitory is being improved constantly, and efforts are made to create a suitable environment for studying and living[9]. In recent years, domestic furniture for college students' dormitory has been systematically studied. Most of them focus on the improvement of basic functions such as sleep, learning and storage, and furniture design based on ergonomics. Compared with the past, there are already many progress, however the dormitory space still has many issues, such as unreasonable layout of furniture, lack of privacy and entertainment, thus the majority of students on the overall satisfaction of the dormitory's furniture is still a negative attitude[10-11].

\section{Dormitory fitness furniture}

Healthy places have a direct impact on college students' fitness motivation, fitness time and fitness projects. For that reason, it is an major factor to find a good place to go to fitness, and it can affect the development of college students after-school fitness activities [12].

Dormitory is the "home" of college students in the school, with living, learning, entertainment and other functions. College students spend their spare time in the dormitory, if they can exercise in the hostel, they can reduce the lower consumption of sports, as well as save the time that spent on the road. At the same time, dormitory exercise is not demanding on the site, facility, and less affected to the outdoor environment. College students can take full advantage of limited time and space for exercise, improve their health and fitness shape [13].

As the furnishings that college students can contact easily during the daily life, the furniture can be used as fitness equipment, which can not only urge people to exercise, but also meet the demand of furniture products. Students will be more active to go to fit with using this convenient and practical fitness equipment. If the dormitory furniture was used as an adjustment of learning exercise, students could be more energetic into the study and research, and be more conducive to develop fitness awareness. Only in that way, can they form a habit of exercise with a clear direction. 


\section{The Demonstration of design}

\subsection{The process of product design}

The design of fitness furniture as the center, and this product combines many researches, such as product appearance, technology, cost and function .etc (Fig. 1).

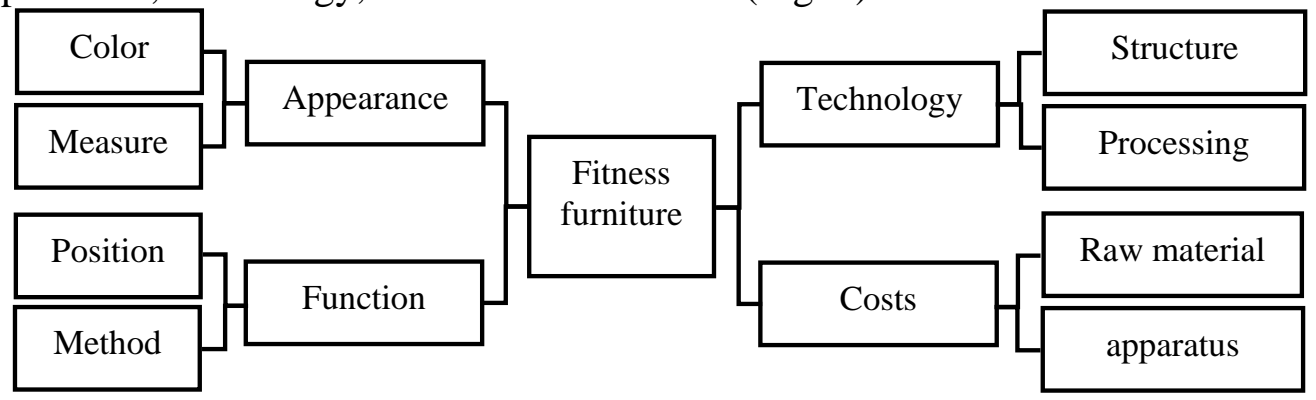

Fig. 1 System structure of product design

\subsection{Analysis of product design}

Most dormitory furniture is double-deck bed of the upper bed and the lower table, and structure of which could offer more storage space. The privacy of students is need for sleeping, learning, and storage function. but the rigid shape of the dormitory furniture to meet the production requirements by manufacture factory result in the lack of fitness function. In order to create a better living conditions, in the design process, we require to retain its advantages, while improving the privacy, functionality, and aesthetics, so that we can design an easy to use, as well as convenient to carry out the fitness system of the new dormitory fitness furniture.

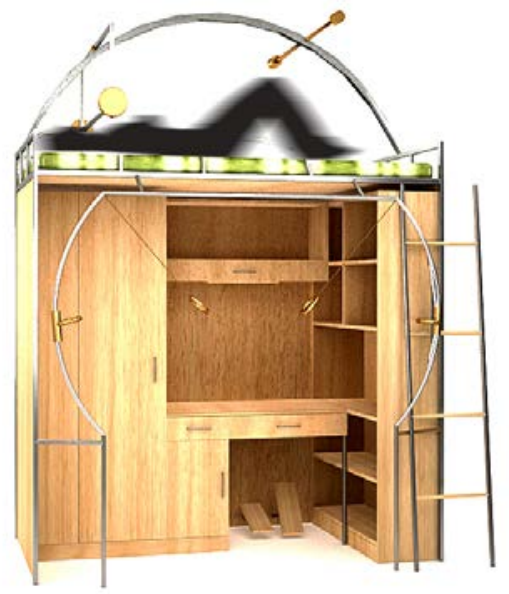

Fig. 2 Weightlifting

5.2.1 The design of movable bed frame

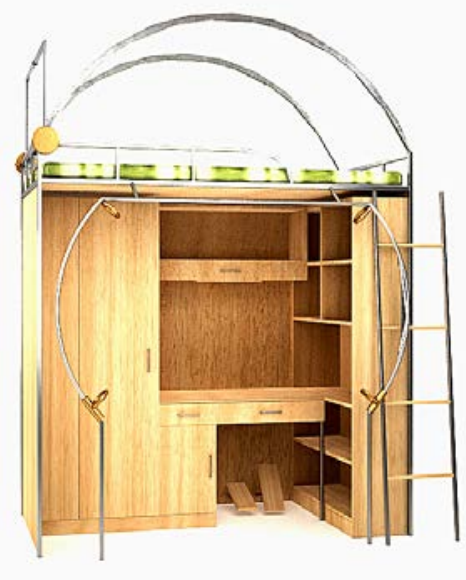

Fig. 4 Frame

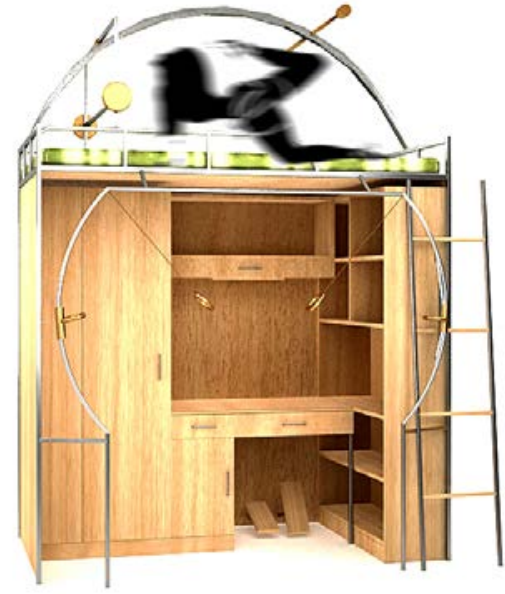

Fig. 3 Cycling

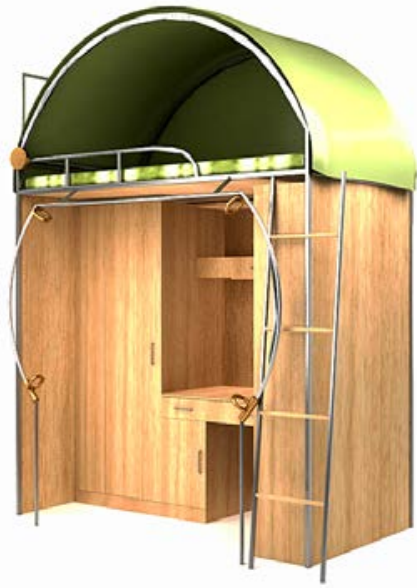

Fig. 5 Mosquito net 
The movable bed frame consists of two parts: the frame part and the arch part. After moving the frame part to the certain position along the horizontal slide rail, the frame and the arch frame are fixed, which ensures the stability of the parts and the safety of the moving process. At this time, gravity weights will slide along with the vertical slide, and they can lie on the bed and do the weightlifting. When stop movement, the gravity weights will return to the end of the bed, thus the frame does not take up any space, and will have a good security (Fig. 2).

Arch components can be moved along the rails on both sides of the bed, and there are damping foot components near the end of the bed. The arch fixed in the middle position, while people can lie on the bed for the supine bicycle movement. Our posture is more standard when we have fitness-assisted (Fig. 3). The arch can be longitudinal separated into two parts, which are fixed on each side of the bed. While it is used as a frame of mosquito net, the bed would be elegant and privacy (Fig. 4, Fig. 5).

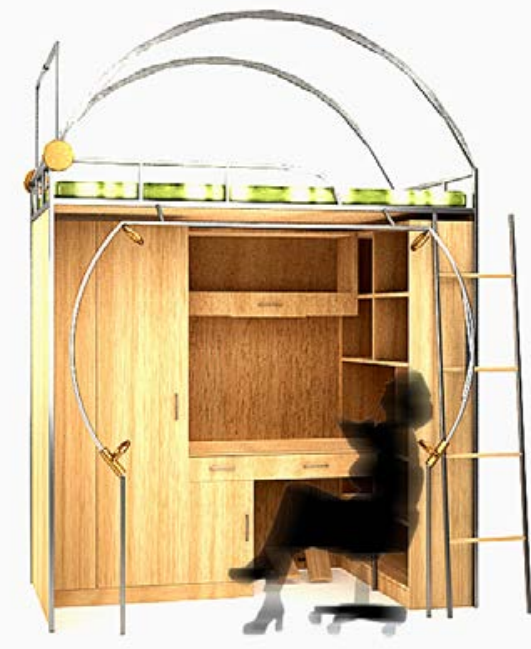

Fig. 6 Pedal

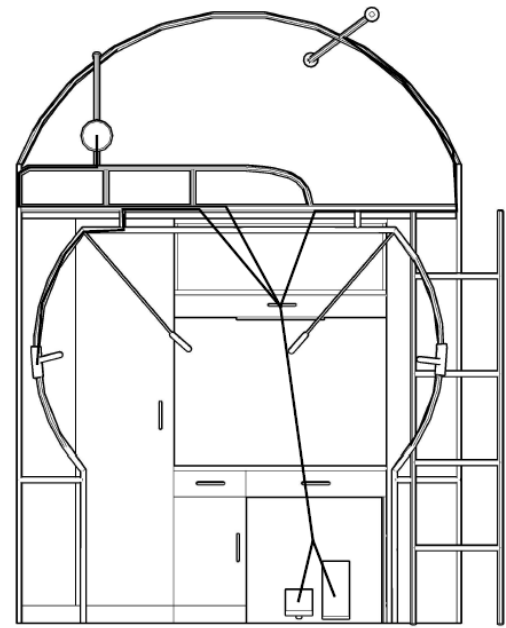

Fig. 7 Storage system

\subsubsection{Fitness pedal design}

Fitness pedal is located under the desk. When we use it, our feet are on the pedal, and alternating stampede it. It can avoid the blood circulation blocking which is caused by the pressure of the sedentary blood vessels through simple movements, thus we can reduce learning pressure after a long learning period, and give a brief relaxation (Fig. 6).

\subsubsection{The Design of Fitness Arches}

Arch component is divided into upper and lower parts, the upper is a straight tube fixed at the edge of the bed, which can be carried out such as fitness, exercise, upper arm muscles. There are two steel pipes made from curved mutual symmetry in the lower part, and the bottom of them equipped the weight of gravity. When we hold the handle, we can straighten the arm and push it up to exercise the deltoid. The ring at the top of the bend is connected with the weight, if the person standing in the middle, and put the ring down, they can exercise the chest and the muscles of the upper arm (Fig. 8, Fig. 9). 


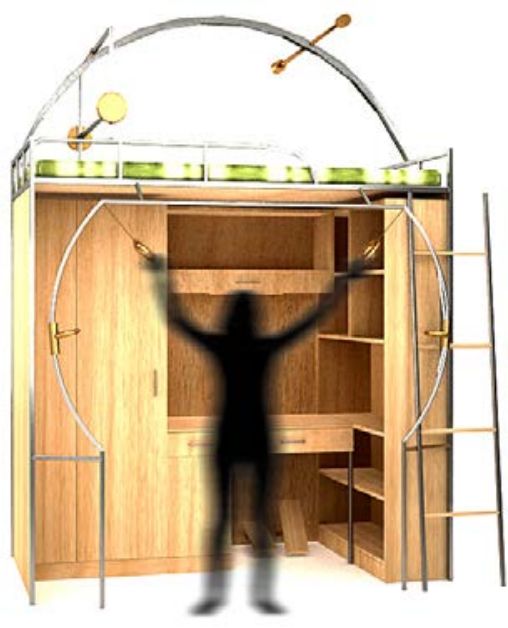

Fig. 8 Pull down motion

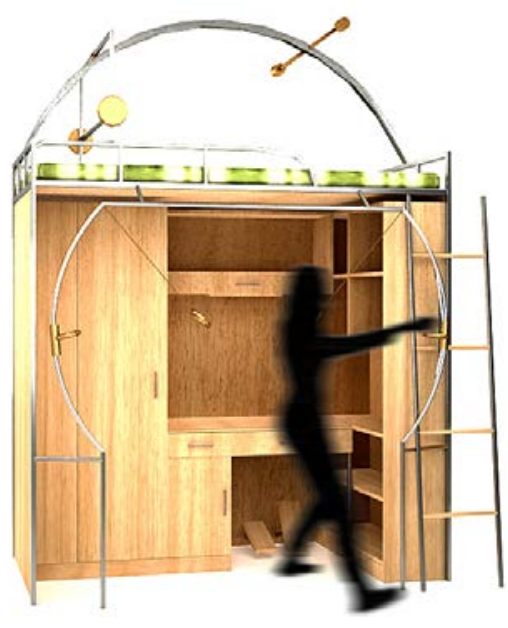

Fig. 9 Push up motion

\subsubsection{The Design of Energy system}

In the design of weightlifting equipment, supine bicycle equipment, push up equipment, pull down equipment and arch component, there are power generation devices. The kinetic energy during exercise is converted to electrical energy, which is stored in the battery. The electrical can provide energy to small lamps or small power storage equipment. This design can not only save energy, but also bring the power to them to exercise (Fig. 7).

This set of design can retain the storage, learning, sleeping and other basic functions, while the design of mosquito nets will protect the privacy of students. There is a variety of fitness equipment, so the students will get a systemic exercise with it. Therefore it is a set of practical fitness as a whole of the multi-functional dormitory furniture.

\section{Product design evaluation}

The construction of index system is in accordance with the principle of design. It will establish evaluation structure from the four dimensions of art, technology, economy, function, and then comprehensive four dimensions of the evaluation of the general product. We evaluate contents of choice should not only consider the general evaluation criteria of four dimensions, but also should pay attention to choosing an appropriate view of evaluation. Thus we can establish an evaluation system which from the user to the enterprise to the society; from the visual, tactile sensation to the psychological, cultural; from the function to the material to the process, from all aspects of the evaluation system of cost to the benefit of multiple levels (Table 1).

Table 1 Multidimensional product evaluation

\begin{tabular}{|c|c|c|}
\hline $\begin{array}{l}\text { Evaluation } \\
\text { dimension }\end{array}$ & $\begin{array}{l}\text { Evaluation } \\
\text { method }\end{array}$ & Effect \\
\hline Artistry & Subjective qualitative & $\begin{array}{l}\text { Through the user needs and aesthetic, } \\
\text { positioning the product of the art }\end{array}$ \\
\hline Technology & $\begin{array}{l}\text { Qualitative and } \\
\text { quantitative } \\
\text { combination }\end{array}$ & $\begin{array}{l}\text { Analysis of product structure, process, } \\
\text { selection of technical priorities }\end{array}$ \\
\hline Economy & Quantitative evaluation & $\begin{array}{l}\text { Product estimation and evaluation } \\
\text { by product cost estimation and sales volume statistics }\end{array}$ \\
\hline Function & $\begin{array}{l}\text { Qualitative and } \\
\text { quantitative } \\
\text { combination }\end{array}$ & $\begin{array}{c}\text { Statistical user requirements and product performance } \\
\text { and customer satisfaction for product function } \\
\text { optimization evaluation }\end{array}$ \\
\hline
\end{tabular}




\section{Summary}

Dormitory is the habitat for students to study and live. Provided that we combine the furniture of the dormitory and the fitness equipment, we can both save space and make full use of spare time. In that case, students can promote the awareness of bodybuilding and make the fitness more active and scientific. At the same time, it could hold back the trend of college students' physique declining. The design of dormitory fitness furniture is of great significance in ensuring the health of college students and promoting the movement of them. Therefore, it is necessary to take a fitness function into consideration in the future of furniture design.

At present, there are no relatively mature and stable industrial systems in the development and research of the fitness furniture, which basically stay in the theoretical stage and small scale production. Lacking in experience, technology and equipment, the fitness furniture designers will find it is full of challenge. However, with the development of science and the desire for fitness in all industries, the designers are sure to overcome all the difficulties and bring us the fitness furniture which is practical, safe, good looking and easy to use.

\section{Acknowledgments}

The project was supported by Key Laboratory of Wood Industry and Furniture Engineering of Sich uan Provincial Colleges and Universities, Sichuan Provincial Philosophy Social Sciences Key Rese arch Base of Modern Design and Culture Research Center (Grant No. MD15E017), Sichuan Provin cial Key Research Base of Philosophy and Social Science-Yi Culture Research Center (Grant No. Y ZWH1433).

\section{References}

[1] Tian Huaxi, Liu Hangwei, On the Feasibility and Importance of Dormitory Fitness for Undergraduates, Sports World (Academic Edition),2014, pp.114-115

[2] Liang Jin, Guan Jichun, Li jun, Investigation and Analysis of Popular Exercice Methods Used in China, Journal of Tianjin Institute physical Education,1997,12, pp.44-47

[3] Hai Liujuan, A Study on the Relationship of College students' Physical exercise, Resilience and Well-being, Master's thesis of Nanchang University(2012)

[4] Lin Shaoqin, Yu Furong, Analyzing the Fitness Needs of the Independent College Students in China, Sports Science Research,2012,16, pp.81-84

[5] Pan Ling, Chen Yunhai, Yu Xuenong,etc, Investigation and Analysis of the State of University Students' Physical Activities in Kunming, Journal of Medicine \& Pharmacy of Chinese Minorities,2007, pp.134-135

[6] Chen Meng, Analysis of the Effect of Indoor Fitness on the All - round Development of People under the Background of Urbanization.Master's thesis of Shandong Normal University(2012)

[7] Liu Xueyu, Gu Weinong, Analysis on Higher Vocational College Students Fitness Sports Consumption.Bulletin of Sport Science and Technology, 2012,20, pp.107-109

[8] Chen Xiaomei, Psychological Crisis of College Students and the Relationship of Roommates, Journal of Fujian Commercial College,2011, pp.65-68

[9] Lv Honglin, Talking about the Change of the Dormitory Space Design in Today 's College, Technology Wind,2010, pp.14

[10]Feng Xiujuan, The Study of College Students' Dormitory Furniture Design Based on the Humanized Concept, Master’s thesis of Northeast Forestry University(2015) 
[11]Luo Shan, A study On the Design of College Students' Dormitory Furniture, Journal of Nanping Teachers College,2013,32, pp.79-80

[12]He Haiqiang, Zhou Yali, Research on Fitness Activities and the Health Investment of Female Students of Colleges and Universities in Hunan Province, Sport Science and Technology,2013,34, pp.84-86

[13]Tang Xiaoyi, Zhou Yali, Exercise Prescription Design of Practical Dormitory for Postgraduates, Chinese School Sports,2009, pp.52-53 